\title{
Fault Diagnosis of Power Systems Using Intuitionistic Fuzzy Spiking Neural P Systems
}

\author{
Hong Peng, Jun Wang, Jun Ming, Peng Shi, Fellow, IEEE, Mario J. Pérez-Jiménez, \\ Wenping Yu, and Chengyu Tao
}

\begin{abstract}
In this paper, intuitionistic fuzzy spiking neural $P$ (IFSNP) systems as a variant are proposed by integrating intuitionistic fuzzy logic into original spiking neural $\mathbf{P}$ systems. Compared with a common fuzzy set, intuitionistic fuzzy set can more finely describe the uncertainty due to its membership and non-membership degrees. Therefore, IFSNP systems are very suitable to deal with fault diagnosis of power systems, specially with incomplete and uncertain alarm messages. The fault modeling method and fuzzy reasoning algorithm based on IFSNP systems are discussed. Two examples are used to demonstrate the availability and effectiveness of IFSNP systems for fault diagnosis of power systems. Case studies involve single fault, complex fault, and multiple faults with protection device failures and incorrect tripping signals.
\end{abstract}

Index Terms-Power systems, fault diagnosis, spiking neural $P$ systems, intuitionistic fuzzy set.

\section{INTRODUCTION}

$\mathbf{T}$ HE POWER system consists of many system elements, such as generators, transformers, bus bars and transmission lines, which are protected by protection systems comprised of protective relays (PRs), circuit breakers (CRs) and communication equipment. The supervisory control and data acquisition (SCADA) system is equipped together with electric power systems. Fault diagnosis of power systems is a process of discriminating the faulted system elements by tripping of protective relays and circuit breakers. When a fault event occurs, it can lead to a large amount of alarm messages in SCADA system. The alarm messages must be analyzed by dispatchers according to their operating experiences in order to

This work was supported in part by the National Natural Science Foundation of China under Grant 61472328, in part by the Chunhui Project Foundation of the Education Department of China under Grant Z2016143 and Grant Z2016148, and in part by the Research Foundation of the Education Department of Sichuan Province, China under Grant 17TD0034. Paper no. TSG-01301-2016.

H. Peng is with the School of Computer and Software Engineering, Xihua University, Chengdu 610039, China (e-mail: ph.xhu@hotmail.com).

J. Wang, J. Ming, W. Yu, and C. Tao are with the School of Electrical and Information Engineering, Xihua University, Chengdu 610039, China (e-mail wangjun@mail.xhu.edu.cn).

P. Shi is with the School of Electrical and Electronic Engineering, University of Adelaide, Adelaide, SA 5005, Australia (e-mail: peng.shi@adelaide.edu.au).

M. J. Pérez-Jiménez is with the Research Group of Natural Computing, Department of Computer Science and Artificial Intelligence, University of Seville, 41012 Sevilla, Spain (e-mail: marper@us.es). identify the faults. However, the received data is often incomplete and tripping of protective relays and circuit breakers is sometime uncertain. Therefore, fault diagnosis is a difficult and complicated task since circuit breakers may fail to operate the multiple faults with the incomplete and uncertain alarm messages.

The expert systems (ES)-based methods have been used to deal with fault diagnosis of power systems [1]-[3]. The ESbased methods are suitable for operating logics of protective relays and circuit breakers as well as the diagnosis experience of operators. However, main drawbacks of the ES-based methods are the incapacity of generalization and the difficulty of validating and maintaining large rule base. With their attractive features, artificial neural networks (ANNs)-based systems have been employed as an intelligent fault diagnosis tool [4]-[6]. Nonetheless, most of the ANN-based diagnosis systems suffer from the "black-box" phenomenon since it is difficult to extract domain knowledge encoded in a trained network to explain its results intuitively. In addition, the performance of ANN-based diagnosis systems is highly restricted without the extensive confirmation of the quality of training process and the quantity of training samples. The fault diagnosis of power systems can be also formulated as an optimization problem. Some optimization techniques, such as genetic algorithms (GAs) [7], Honey-Bee Mating Optimization (HBMO) [8] and artificial bee colony (ABC) [9], were employed to solve the optimization problem. Since the outage area must be identified initially, the loss of a boundary CB alarm may lead to the failure of such methods. In fault diagnosis of power systems, a key problem is how to handle the incomplete and uncertain alarm messages of tripping of circuit breakers. Fuzzy logic provides a more usable and accessible technique to model the inaccuracy and uncertainty in fault diagnosis. Some techniques that incorporate fuzzy logic have been developed for fault diagnosis of power systems, for example, fuzzy logic (FL) [10], fuzzy relation (FR) [11] and fuzzy digraph models (FDM) [12]. Petri nets (PNs) are a useful tool for event modeling in a concurrent structure. However, it lacks the ability to handle uncertainty. Thus, fuzzy Petri nets (FPNs) [13], [14] that combine fuzzy logic with PNs have been employed to deal with the uncertainty existing in the operation of protective devices.

Membrane computing is a class of distributed parallel computing models inspired from the structure and functioning of living cells as well as interaction of living cells in tissues and organs, known as P systems [15], [16]. In past years, a various 
of $\mathrm{P}$ systems and variants have been proposed and applied in a lot of real-world problems [17]-[27]. Spiking neural P systems (in short, SNP systems) are one of main forms of $\mathrm{P}$ systems. A SNP system can be viewed as a directed graph whose arcs represent the synaptic connections among the neurons [16], [28]-[30]. In recent years, a class of variants, which integrate different fuzzy logics in SNP systems, were developed, called the fuzzy spiking neural P system (in short, FSNP systems) [31]-[34]. Furthermore, FSNP systems have been used to deal with fault diagnosis of power systems [35]-[37]. Intuitionistic fuzzy set (IFS) has been proposed to deal with more finely the incompleteness and uncertainty [38]-[40]. IFS, which is a natural generalization of usual fuzzy set, seems to be useful in modeling many real life situations. IFS can finely characterize the membership level of an element $x$ to fuzzy set $A$ by providing two measures (membership and nonmembership degrees) simultaneously. However, IFS has not been used to handle fault diagnosis problem of power systems.

In this paper a new variant is proposed by integrating IFS in SNP systems, called intuitionistic fuzzy spiking neural $\mathrm{P}$ systems (in short, IFSNP systems). The fault diagnosis model based on IFSNP systems is discussed in detail. Main contribution of this paper stays on proposing the IFSNP systems and developing a novel modeling method for fault diagnosis of power systems. Compared with the existing FSNP systems, differences of IFSNP systems include: (1) intuitionistic fuzzy number (IFN) is used to express alarm information and imprecise knowledge in fault diagnosis problems of power systems; (2) fuzzy reasoning mechanism of IFSNP systems is based on intuitionistic fuzzy logic; (3) diagnosis result (whether an element is a fault in a section) is described by a membership degree and a non-membership degree simultaneously. Therefore, the proposed IFSNP systems can better model the imperfect information, especially under imperfectly defined alarm information and imprecise knowledge in fault diagnosis of power systems.

The remainder of this paper is organized as follows. IFSNP systems are discussed in Section II, including the definition, modeling and reasoning methods. Three case studies of power systems with different structures are provided in Section III. Conclusions are finally drawn in Section IV.

\section{IFSNP SYSTEMS}

\section{A. Definition}

Let $X$ be a universe of discourse. Intuitionistic fuzzy set (IFS) is a generalized fuzzy set introduced by Atanassov [38], shown as follows:

$$
A=\left\{<x, \mu_{A}(x), v_{A}(x)>\mid x \in X\right\}
$$

which is characterized by a membership function $\mu_{A}: X \rightarrow$ $[0,1]$ and a non-membership function $v_{A}: X \rightarrow[0,1]$, with the condition

$$
0 \leq \mu_{A}(x)+v_{A}(x) \leq 1, \quad \forall x \in X
$$

where the numbers $\mu_{A}(x)$ and $\nu_{A}(x)$ denote the membership and non-membership degrees of the element $x$ to the $A$, respectively.
For each IFS $A$ in $X$, if $\pi_{A}(x)=1-\mu_{A}(x)-v_{A}(x)$, then $\pi_{A}(x)$ is called the indeterminacy degree or hesitation degree of $x$ to $A$. Specially, if $\pi_{A}(x)=1-\mu_{A}(x)-v_{A}(x)=0, \forall x \in X$, then the IFS $A$ is reduced to a common fuzzy set.

For convenience, we call $\alpha=\left(\mu_{\alpha}, v_{\alpha}\right)$ an intuitionistic fuzzy number (IFN), where $\mu_{\alpha} \in[0,1], v_{\alpha} \in[0,1]$, and $\mu_{\alpha}+v_{\alpha} \leq 1$.

Let $\alpha=\left(\mu_{\alpha}, v_{\alpha}\right)$ and $\beta=\left(\mu_{\beta}, v_{\beta}\right)$ be two intuitionistic fuzzy numbers, and $\lambda$ is a real number in $[0,1]$. Three operations are introduced as follows:

(1) $\alpha \oplus \beta=\left(\max \left(\mu_{\alpha}, \mu_{\beta}\right), \min \left(v_{\alpha}, v_{\beta}\right)\right)$;

(2) $\alpha \otimes \beta=\left(\mu_{\alpha} \cdot \mu_{\beta}, v_{\alpha}+v_{\beta}-v_{\alpha} \cdot v_{\beta}\right)$;

(3) $\lambda \alpha=\left(\lambda \mu_{\alpha}, \lambda v_{\alpha}\right)$.

Let $S(\alpha)=\mu_{\alpha}-v_{\alpha}$ and $H(\alpha)=\mu_{\alpha}+v_{\alpha}$. For $\alpha$ and $\beta$, $\alpha<\beta$ if and only if (1) $S(\alpha)<S(\beta)$, or (2) $S(\alpha)=S(\beta)$ and $H(\alpha)=H(\beta)$.

Definition 1: An intuitionistic fuzzy spiking neural $\mathrm{P}$ system (IFSNP system, in short) of degree $m$ is a construct

$$
\Pi=\left(A, \sigma_{1}, \sigma_{2}, \ldots, \sigma_{m}, s y n, I, O\right)
$$

where:

(1) $A=\{a\}$ is the singleton alphabet ( $a$ denotes spike);

(2) $\sigma_{1}, \sigma_{2}, \ldots, \sigma_{m}$ are neurons of the form $\sigma_{i}=\left(\alpha_{i}, \tau_{i}, r_{i}\right)$, $i \in\{1,2, \ldots, m\}$ where:

(a) $\sigma_{i}$ is an intuitionistic fuzzy number, denoting the initial value of spikes contained in $\sigma_{i}$;

(b) $\tau_{i}$ is a real number in $[0,1]$, denoting the confidence level associated with the neuron;

(c) $r_{i}$ is a firing rule/spiking rule, of the form $a^{\alpha} \rightarrow a^{\alpha}$ or $a^{\alpha} \rightarrow a^{\beta}$, where $\alpha, \beta$ are two intuitionistic fuzzy numbers;

(3) syn $\subseteq\{1,2, \ldots, m\} \times\{1,2, \ldots, m\}$, with $(i, i) \notin s y n$ for $\forall 1 \leq i \leq m$ is the synapse graph, defining the synapses among neurons;

(4) $I$ and $O$ denote the sets of input neurons and output neurons, respectively.

IFSNP systems are a variant of original SNP systems, which integrate intuitionistic fuzzy logic into their mechanisms. The firing mechanism of neurons can be described as follows: for a neuron $\sigma_{i}$, if its spiking rule is enabled, then the neuron fires and its spike value $\alpha$ is consumed, and then a spike with value $\beta$ is generated; once the spike with value $\beta$ is emitted, all successor neurons (with $(i, j) \in$ syn) will receive the spike.

\section{B. Modeling and Fuzzy Reasoning}

In many applications fuzzy production rules have been commonly used in knowledge representation, where their antecedent and consequent use "AND" and "OR" operations to connect multiple propositions respectively. The following two types of fuzzy production rules have been used to construct fuzzy knowledge base:

Type 1: IF $p_{1}$ AND $p_{2}$ AND $\ldots$ AND $p_{k-1}$ THEN $p_{k}$ $(\mathrm{CF}=\tau)$

Type 2: if $p_{1}$ OR $p_{2}$ OR $\ldots$ OR $p_{k-1}$ THEN $p_{k}(\mathrm{CF}=\tau)$ where $p_{1}, p_{2}, \ldots, p_{k-1}, p_{k}$ are $k$ propositions, and $\tau$ is a real number in $[0,1]$ and denotes the confidence factor $(\mathrm{CF})$ of the fuzzy production rule. 


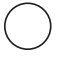

(a)

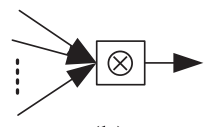

(b)

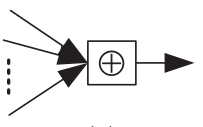

(c)
Fig. 1. Three types of neurons: (a) proposition neuron, (b) $\otimes$-type rule neuron and (c) $\oplus$-type rule neuron.

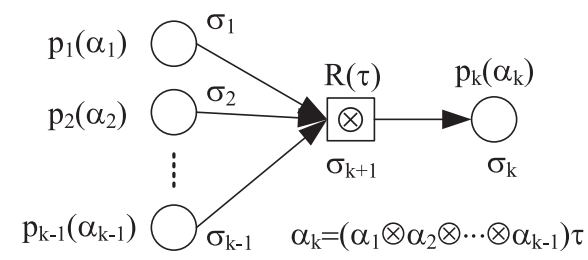

Fig. 2. Modeling type 1-fuzzy production rule based on IFSNP systems.

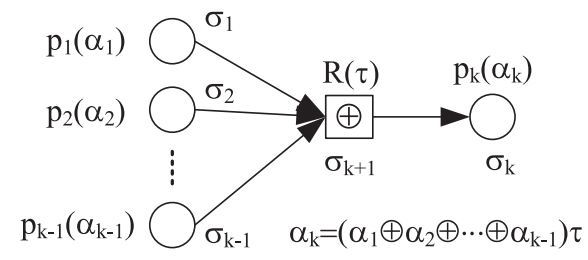

Fig. 3. Modeling type 2-fuzzy production rule based on IFSNP systems.

To model the fuzzy production rules, the neurons in IFSNP systems are further classified into three classes: proposition neurons, $\oplus$-type rule neurons and $\otimes$-type rule neurons. Proposition neurons are used to characterize fuzzy propositions in a fuzzy knowledge base. $\otimes$ - and $\oplus$-type rule neurons are used to denote "AND"- and "OR"-type fuzzy production rules, respectively. Fig. 1 shows the three types of neurons.

A type 1-fuzzy production rule can be modeled by an IFSNP system, shown in Fig. 2. The reasoning procedure of IFSNP system can be described as follows. Initially, proposition neuron $\sigma_{i}$ is assigned a spike with value $\alpha_{i}, i=1,2, \ldots, k-1$. Thus, the neurons fire and each emit a spike with value $\alpha_{1}, \alpha_{2}, \ldots, \alpha_{k-1}$, respectively. Afterward, $\otimes$-type rule neuron $\sigma_{k+1}$ receives $k-1$ spikes with value $\alpha_{k+1}=\alpha_{1} \otimes \alpha_{2} \otimes$ $\ldots \otimes \alpha_{k-1}$. Then, rule neuron $\sigma_{k+1}$ fires and emits a spike (with value $\alpha_{k+1} \tau$ ) to the subsequent proposition neuron $\sigma_{k}$. Finally, neuron $\sigma_{k}$ receives the spike. Therefore, computing result of the system is $\alpha_{k}=\left(\alpha_{1} \otimes \alpha_{2} \otimes \cdots \otimes \alpha_{k-1}\right) \tau$.

Fig. 3 shows another IFSNP system used to model a type 2-fuzzy production rule. The reasoning procedure of the IFSNP system can be described as follows. Initially, proposition neurons $\sigma_{1}, \sigma_{2}, \ldots, \sigma_{k-1}$ are each assigned a spike, with values $\alpha_{1}, \alpha_{2}, \ldots, \alpha_{k-1}$, respectively. Thus, the neurons fire and each emit a spike with value $\alpha_{1}, \alpha_{2}, \ldots, \alpha_{k-1}$, respectively. Afterward, $\oplus$-type rule neuron $\sigma_{k+1}$ receives $k-1$ spikes with value $\alpha_{k+1}=\alpha_{1} \oplus \alpha_{2} \oplus \ldots \oplus \alpha_{k-1}$. Then, rule neuron $\sigma_{k+1}$ fires and emits a spike (with value $\alpha_{k+1} \tau$ ) to the subsequent proposition neuron $\sigma_{k}$. Finally, neuron $\sigma_{k}$ receives the spike. Therefore, computing result of the system is $\alpha_{k}=\left(\alpha_{1} \oplus \alpha_{2} \oplus \ldots \oplus \alpha_{k-1}\right) \tau$.

In the following, we describe the proposed fuzzy reasoning algorithm based on IFSNP systems. Suppose that the
TABLE I

FuZZy REASONING ALGORITHM BASED ON IFSNP SYSTEMS

\begin{tabular}{|l|}
\hline Input: $\Pi, \alpha_{i}$ for $\forall \sigma_{i} \in I$ \\
\hline Output: $\alpha_{i}$ for $\forall \sigma_{i} \in O$; \\
\hline Begin \\
(1) Read $C, D_{1}, D_{2}, E, \delta_{0}$ and $\theta_{0}$ \\
(2) $t \leftarrow 1$; \\
(3) while (halting condition is not met) \\
(4) Proposition neurons evaluate and fire; \\
(5) $\quad \delta_{t} \leftarrow\left(D_{1}^{T} \otimes \theta_{t-1}\right)+\left(D_{2}^{T} \oplus \theta_{t-1}\right)$ \\
(6) $\quad$ Rule neurons evaluate and fire; \\
(7) $\quad \theta_{t} \leftarrow E^{T} \oplus\left(C \odot \delta_{t}\right)$ \\
(8) $\quad t \leftarrow t+1$; \\
(9) endwhile \\
(10) Export all $\alpha_{i}$ of $\sigma_{i}$ in $O$; \\
End
\end{tabular}

considered IFSNP system $\Pi$ contains $m$ proposition neurons and $n$ rule neurons $(\oplus$-type or $\otimes$-type). For convenience, several notions and operations are firstly introduced as follows.

(1) Vector $\theta=\left(\theta_{1}, \theta_{2}, \ldots, \theta_{m}\right)$ denotes the values of spikes in the $m$ proposition neurons, where $\theta_{i}$ is an intuitionistic fuzzy number, $1 \leq i \leq m$.

(2) Vector $\delta=\left(\delta_{1}, \delta_{2}, \ldots, \delta_{n}\right)$ denotes the values of spikes in the $n$ rule neurons, where $\delta_{i}$ is an intuitionistic fuzzy number, $1 \leq i \leq n$.

(3) Matrix $C=\operatorname{diag}\left(c_{1}, c_{2}, \ldots, c_{n}\right)$ is called the confidence matrix, where $c_{i} \in[0,1]$ denotes confidence factor (CF) of $i$-th fuzzy production rule, $1 \leq i \leq n$.

(4) Matrix $D_{1}=\left(d_{i j}\right)_{m \times n}$ denotes the synapse connection from proposition neurons to $\otimes$-type rule neurons. If there is a directed arc from proposition neuron $\sigma_{i}$ to $\otimes$-type rule neuron $\sigma_{j}$, then $d_{i j}=1$; otherwise $d_{i j}=0,1 \leq i \leq m, 1 \leq j \leq n$.

(5) Matrix $D_{2}=\left(d_{i j}\right)_{m \times n}$ denotes the synapse connection from proposition neurons to $\oplus$-type rule neurons. If there is a directed arc from proposition neuron $\sigma_{i}$ to $\oplus$-type rule neuron $\sigma_{j}$, then $d_{i j}=1$; otherwise $d_{i j}=0,1 \leq i \leq m, 1 \leq j \leq n$.

(6) Matrix $E=\left(e_{j i}\right)_{n \times m}$ denotes the synapse connection from rule neurons to proposition neurons. If there is a directed arc from rule neuron $\sigma_{j}$ to proposition neuron $\sigma_{i}$, then $e_{j i}=1$; otherwise $e_{j i}=0,1 \leq j \leq n, 1 \leq i \leq m$.

(7) $C \odot \delta=\left(c_{1} \delta_{1}, c_{2} \delta_{2}, \ldots, c_{n} \delta_{n}\right)^{T}$.

(8) $D \otimes \theta=\left(\tilde{d}_{1}, \tilde{d}_{2}, \ldots, \tilde{d}_{n}\right)^{T}$, where $\tilde{d}_{j}=d_{1 j} \theta_{1} \otimes d_{2 j}$ $\theta_{2} \otimes \ldots \otimes d_{m j} \theta_{m}, j=1,2, \ldots, n$.

(9) $D \oplus \theta=\left(\tilde{d}_{1}, \tilde{d}_{2}, \ldots, \tilde{d}_{n}\right)^{T}$, where $\tilde{d}_{j}=d_{1 j} \theta_{1} \oplus d_{2 j}$ $\theta_{2} \oplus \ldots \oplus d_{m j} \theta_{m}, j=1,2, \ldots, n$.

Based on neuron's firing mechanism in IFSNP systems, fuzzy reasoning algorithm can be summarized in Table I.

\section{CASE Studies}

In this section, two different examples of power systems are used to illustrate and validate the availability and effectiveness of the proposed IFSNP systems: a six-bus $69 \mathrm{kV}$ distribution system and a $345 \mathrm{kV}$ transmission system. In the two examples, several cases are discussed, including single fault, complex fault and multiple faults. The diagnosis results of the proposed method are compared with other diagnosis methods. 
TABLE II

LINGUISTIC TERMS AND THE CORRESPONDING INTUITIONISTIC FUZZY NUMBERS (IFNS)

\begin{tabular}{|c|c|}
\hline Linguistic terms & Intuitionistic Fuzzy Numbers (IFNs) \\
\hline Extremely high (EH) & {$[1.00,0.00]$} \\
Very very high (VVH) & {$[0.90,0.10]$} \\
Very high (VH) & {$[0.80,0.10]$} \\
High (H) & {$[0.70,0.20]$} \\
Medium high (MH) & {$[0.60,0.30]$} \\
Medium (M) & {$[0.50,0.40]$} \\
Medium low (ML) & {$[0.40,0.50]$} \\
Low (L) & {$[0.25,0.60]$} \\
Very low (VL) & {$[0.10,0.75]$} \\
Very very low (VVL) & {$[0.10,0.90]$} \\
Extremely low (EL) & {$[0.00,1.00]$} \\
\hline
\end{tabular}

TABLE III

The Confidence Degrees of the Operated Protective Devices

\begin{tabular}{|c|cc|cc|cc|}
\hline \multirow{2}{*}{ Sections } & \multicolumn{6}{|c|}{ Protective devices } \\
\cline { 2 - 7 } & \multicolumn{2}{|c|}{ Main } & \multicolumn{2}{|c|}{ Nearby } & \multicolumn{2}{c|}{ Remote } \\
\cline { 2 - 7 } & Relays & CBs & Relays & CBs & Relays & CBs \\
\hline L & VVH & VVH & VH & VH & H & H \\
B & VVH & VVH & - & - & H & H \\
T & VVH & VVH & VH & VH & H & H \\
\hline
\end{tabular}

\section{A. Fault Diagnosis Model Based on IFSNP Systems}

In this work, IFSNP systems are used to diagnose the faults of main sections, including transmission line, bus and transformer. In IFSNP systems, proposition neurons and rule neurons both are used to express the causal relationship between a fault section and its protective devices. The IFN value of proposition neuron is used to express the confidence degree of protective relay/circuit breaker, while rule neuron uses IFN value to express the probability of tripping the circuit breaker by protective operation. Considering the uncertainty of experts and senior dispatchers, fuzzy linguistic terms are used to describe the confidence degrees or probabilities, shown in Table II.

For each suspicious component in outage area, IFSNP systems is used to build its fault diagnosis model. The diagnosis procedure based on IFSNP systems has three steps: (1) retrieve the operational information of each device from SCADA system as the input data of IFSNP systems; (2) use fuzzy reasoning algorithm in Table I to obtain fault confidence levels of suspicious fault components; (3) distinguish the fault components according to the reasoning results.

In the IFSNP systems, the confidence factor (CF) is a real number in $[0,1]$. Based on the experience and protection level, it is considered that the confidence factor of rule neuron associated with both main protective and nearby backup devices is set to be 1.0, and the confidence factor of rule neuron associated with remote backup devices is set to be 0.9. If it involves multiple levels of protections, the certainty factors can be set to the value corresponding to the highest level of protections. At the same time, confidence degree of each protective device is also assigned according to past experience in fault diagnosis of power systems, including line, bus, protective relay
TABLE IV

The Confidence Degrees of the Non-Operated PROTECTIVE DEVICES

\begin{tabular}{|c|cc|cc|cc|}
\hline \multirow{2}{*}{ Sections } & \multicolumn{5}{|c|}{ Protective devices } \\
\cline { 2 - 7 } & \multicolumn{2}{|c|}{ Main } & \multicolumn{2}{c|}{ Nearby } & \multicolumn{2}{c|}{ Remote } \\
\cline { 2 - 7 } & Relays & CBs & Relays & CBs & Relays & CBs \\
\hline L & L & L & L & L & L & L \\
B & ML & L & - & - & ML & L \\
T & ML & L & ML & L & L & L \\
\hline
\end{tabular}

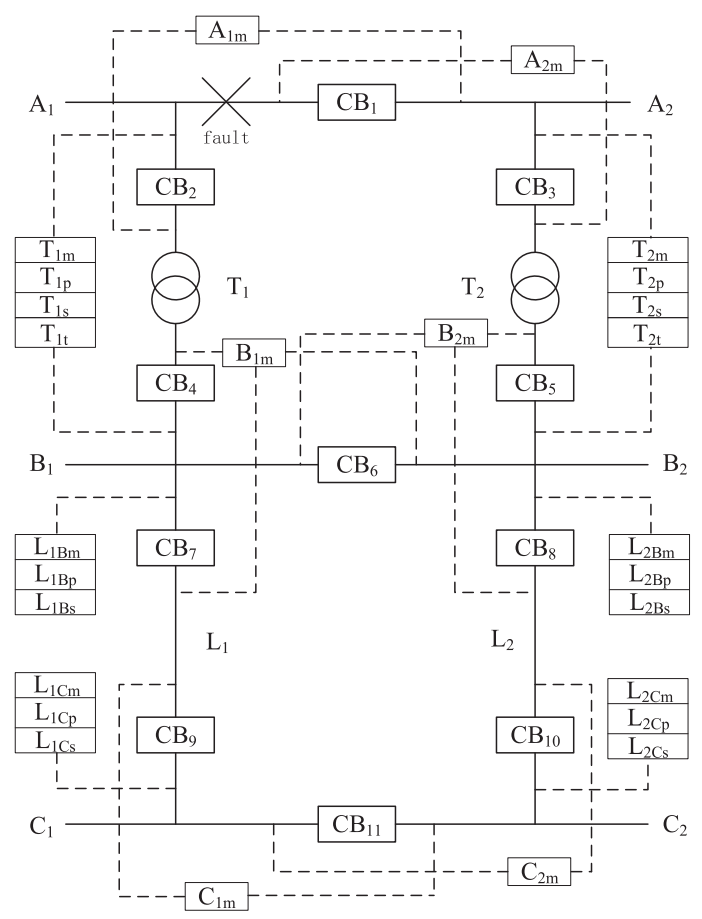

Fig. 4. A six-bus $69 \mathrm{kV}$ distribution system.

and circuit breaker. Tables III and IV provide the confidence degrees of the operated protective devices and non-operated protective devices, respectively. In addition, if the confidence level $\theta$ of a section satisfies the condition $\theta \geq(0.60,0.30)$ the section is a fault; if $\theta \leq(0.40,0.50)$ the section is not a fault; otherwise, it may be a fault.

\section{B. Example I}

The first system studied is a six-bus $69 \mathrm{kV}$ distribution system, shown in Fig. 4, which is adopted from [11]. This system consists of 10 system sections, 10 circuit breakers and 26 protective relays. Symbols are assigned as follows: $A / B / C, L, C B$ and $T$ denote bus, line, circuit breaker and transformer, respectively. The 10 system sections have six buses (labeled by $A_{1}, A_{2}, B_{1}, B_{2}, C_{1}, C_{2}$ ), two transmission lines (labeled by $L_{1}, L_{2}$ ) and two transformers (labeled by $T_{1}, T_{2}$ ). The $10 \mathrm{CBs}$ are labeled as $C B 1, C B 2, \ldots, C B 9, C B 10$. The 26 protective relays are composed of 12 main protective relays (MPR) (labeled by $A_{1 m}, A_{2 m}, B_{1 m}, B_{2 m}$, ), 8 nearby backup relays (labeled by $\left.T_{1 p}, T_{1 s}, T_{1 p}, T_{1 s}, L_{1 B p}, L_{2 B p}, L_{1 C p}, L_{2 C p}\right)$ and 6 remote backup relays (labeled by $T_{1 t}, T_{2 t}, L_{1 B s}, L_{2 B s}, L_{1 C s}, L_{2 C s}$ ). This system 


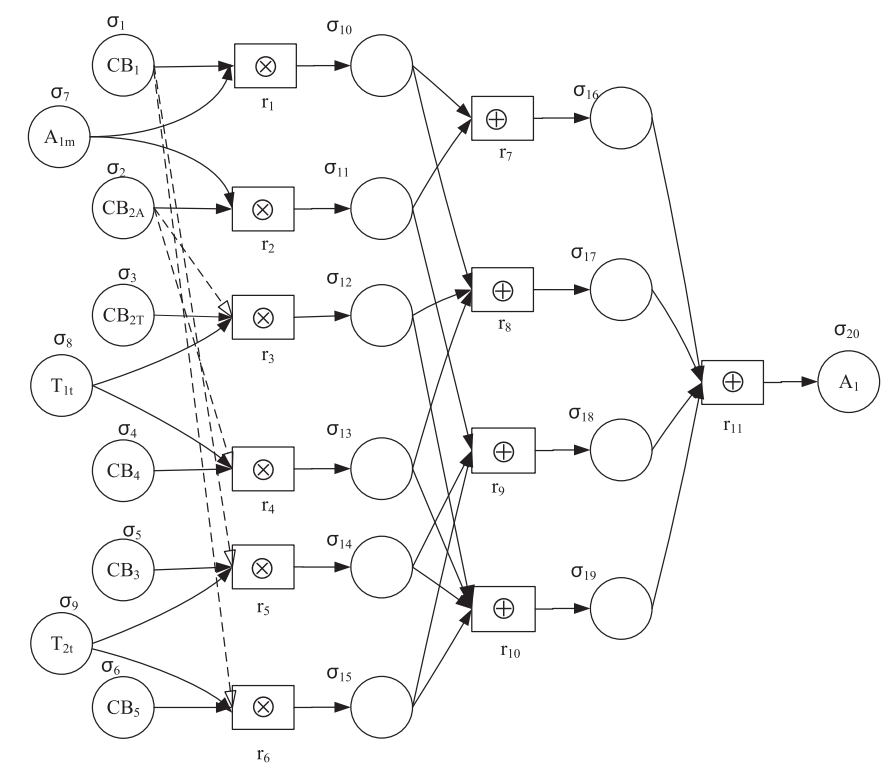

Fig. 5. The fault diagnosis model of bus $A_{1}$ based on IFSNP systems.

was used to test whether the proposed method can diagnose single fault, complex fault and multiple faults with rejection.

The diagnosis model of bus $A_{1}$ can be described by an IFSNP system consisted of 20 proposition neurons and 11 rule neurons, shown in Fig. 5. There are four assistant synapses, including $\left(\sigma_{1}, r_{5}\right),\left(\sigma_{1}, r_{6}\right),\left(\sigma_{2}, r_{3}\right)$ and $\left(\sigma_{2}, r_{4}\right)$, marked by dashed lines with hollow arrow. For clarity, $\left(\sigma_{1}, r_{5}\right)$ is regarded as an example to explain the meaning of these assistant synapses as follows: if $C B_{1}$ successfully opens, then the operation of $T_{2 t}, C B_{3}$ and $C B_{5}$ is invalid, thus their values each are set to be [0.0, 1.0]; otherwise, the operation of $T_{2 t}, C B_{3}$ and $C B_{5}$ is valid.

In the following, three cases are discussed, including single fault, complex fault and multiple faults.

Case 1 (Single Fault Without Failure Devices): Suppose that a fault occurs at the bus $A_{1}$. The fault leads to the operation of main protective relays $A_{1 m}$ and the tripping of circuit breakers $C B_{1}$ and $C B_{2}$ without malfunction and rejection. The information retrieved from SCADA shows the protective relays $A_{1 m}$ operates and circuit breakers $C B_{1}$ and $C B_{2}$ trip.

The fault section can be diagnosed as bus $A_{1}$ by using the IFSNP system in Fig. 5. The proposed fuzzy reasoning algorithm can be used to conclude that output neuron $\sigma_{20}$ has the fuzzy value of $[0.81,0.19](\geq V H)$. Therefore, $A_{1}$ can be recognised as a fault section with the confidence degree 0.81 according to the judgment condition given above. Note that $A_{1}$ is not a fault section only with the credibility of 0.19 . This illustrates that the proposed IFSNP systems can accurately diagnose single fault.

Case 2 (Complex Fault With the Rejection of Circuit Breakers): Suppose that a fault occurs at the bus $A_{1}$. The fault leads to the operation of main protective relays $A_{1 m}$ and trips circuit breakers $C B_{1}$ and $C B_{2}$. But $C B_{2}$ fails to operate, thus the operation of remote backup relays $T_{1 t}$ leads to trip $C B_{2}$ again and $C B_{4}$. The information obtained from SCADA shows that the protective relays $A_{1 m}$ and $T_{1 t}$ operate and circuit breakers $C B_{1}, C B_{2}$ and $C B_{4}$ trip.

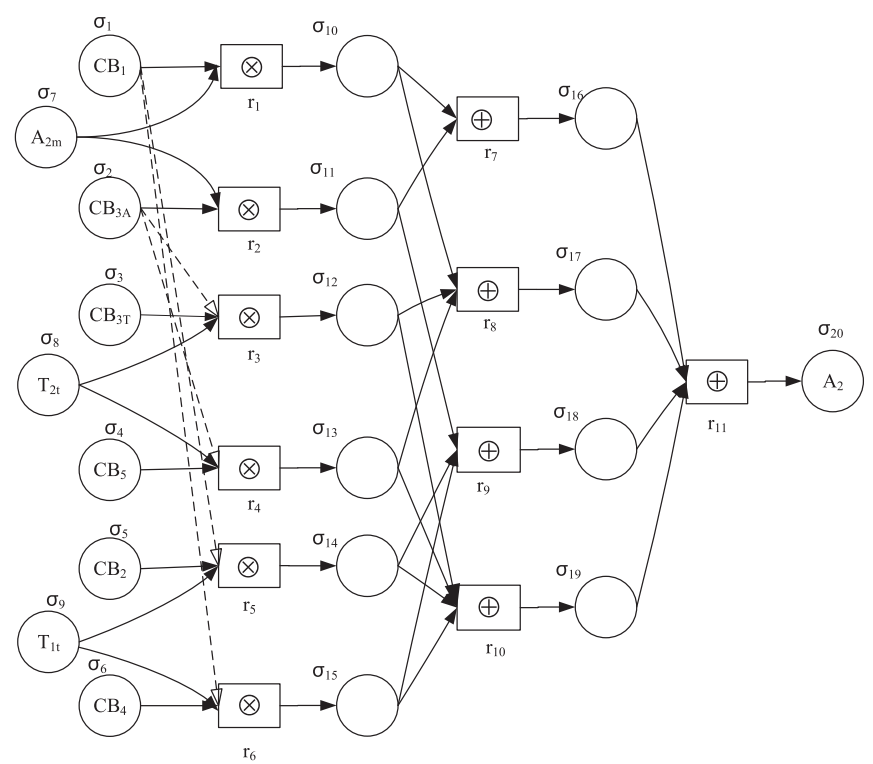

Fig. 6. The fault diagnosis model of bus $A_{2}$ based on IFSNP systems.

Fig. 5 shows the IFSNP system for fault diagnose of $A_{1}$. Based on the IFSNP system, fault diagnosis process of $A_{1}$ can be achieved by the presented fuzzy reasoning algorithm. After fuzzy reasoning, we can obtain that fuzzy value of output neuron $\sigma_{20}$ is $[0.81,0.19]$. Based on the judgment condition, we can judge that $A_{1}$ is a fault section with high confidence degree $(\geq V H)$. Therefore, the proposed method can well distinguish the fault section in the case of complex fault.

Case 3 (Multiple Faults With Rejection of Circuit Breakers): Suppose that multiple faults occur at the buses $A_{1}$ and $A_{2}$. The fault at bus $A_{2}$ leads to the operation of main protective relays $A_{2 m}$ and trips circuit breakers $C B_{1}$ and $C B_{3}$. The fault at bus $A_{1}$ leads to the operation of main protective relays $A_{1 m}$ and trips circuit breakers $C B_{1}$ and $C B_{2}$, but $C B_{2}$ fails to operate. Thus, the operation of remote backup relays $T_{1 t}$ leads to trip $C B_{2}$ again and $C B_{4}$. The information obtained from SCADA indicates that the protective relays $A_{1 m}, A_{2 m}$ and $T_{1 t}$ operate and circuit breakers $C B_{1}, C B_{2}, C B_{3}$ and $C B_{4}$ trip.

The diagnosis models of the multiple faults can be also described by the IFSNP systems in Fig. 5 and Fig. 6, respectively. The presented fuzzy reasoning algorithm is used to conclude the diagnosis result in the case of multiple faults. Since $A_{1}$ and $A_{2}$ have a similar reasoning procedure, the reasoning procedure of bus $A_{1}$ as an example is illustrated as follows.

Initially, $\theta_{0}$ and $\delta_{0}$ can be determined according to the status information of protective relays and circuit breakers in the fault situation and Tables II, III and IV, in which $\theta_{0}$ is a 20-dimensional vector and $\delta_{0}$ is a 11-dimensional vector. The proposed fuzzy reasoning algorithm can be used to conclude that fuzzy value of output neuron $\delta_{20}$ for bus $A_{1}$ is $[0.81,0.19]$. Similarly, we can conclude for bus $A_{2}$ that the fuzzy value of output neurons $\delta_{20}$ is also [0.81, 0.19]. Based on the judgment condition, $A_{1}$ and $A_{2}$ are simultaneously distinguished as the fault sections with high confidence level $(\geq V H)$. Note that the confidence levels of them being not the fault sections are only 0.19 . This indicates that the proposed IFSNP systems are 


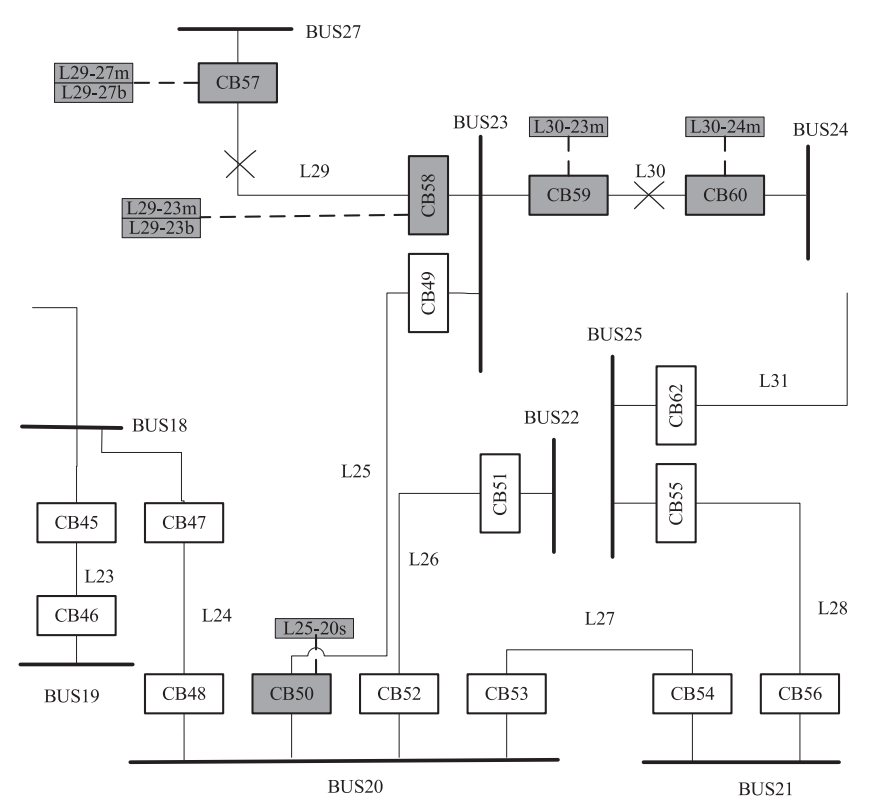

Fig. 7. A $345 \mathrm{kV}$ power transmission system. Here, "m" refers to the main protective relay, "b" denotes the nearby backup relay, and "s" represents the remote backup relay.

suitable to deal with the multiple faults with malfunction and rejection of circuit breakers.

\section{Example II}

The second system studied is a $345 \mathrm{kV}$ power transmission system, shown in Fig. 7, which is adopted from [12]. This system includes 18 system sections, 17 circuit breakers and 60 protective relays. Symbols are assigned as follows: BUS, $\mathrm{L}$ and $\mathrm{CB}$ denote bus, line and circuit breaker, respectively. The 18 system sections include nine buses (labeled by $B U S 18, B U S 19, \ldots, B U S 25, B U S 27)$ and nine transmission lines (labeled by $L 23, L 24, \ldots, L 31$ ). The $17 \mathrm{CBs}$ are labeled as $C B 45, \ldots, C B 60, C B 62$. The 60 protective relays are composed of 26 main protective relays (MPR) (labeled by BUS $18 m, \ldots$, BUS25m,BUS27m, L23 - xm, ..,L31 - xm), 17 nearby backup relays (labeled by $L 23-x b, \ldots, L 31-x b$ ) and 17 remote backup relays (labeled by $L 23-x s, \ldots, L 31-x s$ ). This system was used to test whether the proposed method can diagnose the multiple faults with rejection and incorrect tripping signals.

Case 4 (Multiple Faults With Rejection and Incorrect Tripping Signals): Suppose that multiple faults occur at the transmission line $L 29$ and $L 30$. The fault at line section $L 30$ leads to the operation of main protective relays, $L 30-23 \mathrm{~m}$ and $L 30-24 m$, and the tripping of circuit breakers CB59 and $C B 60$. The fault at line section $L 29$ leads to the operation of main protective relays, $L 29-27 m$ and $L 29-23 m$, but the rejection of $C B 57$ and $C B 58$. Thus, nearby backup relays $L 29-27 b$ and $L 29-23 b$ operate to trip CB57 and CB58. There is also an obscure operation backup relay $L 25-20$ s, which causes $C B 50$ to be tripped. Status information obtained from the SCADA system is as follows: the operated relays are $L 30-23 m, L 30-24 m$, L29-27m,L29-23m, L29 - 27b, $L 29-23 b$ and $L 25-20 s$, and the tripped CBs CB50, CB57, $C B 58, C B 59$ and $C B 60$.
The fault diagnosis models of lines $L 30$ and $L 29$ can be built by two IFSNP systems, shown in Fig. 8 and Fig. 9, respectively. The two IFSNP systems contain each 23 proposition neurons and 13 rule neurons. In the two systems, proposition neurons, $\sigma_{1}, \ldots, \sigma_{10}$ as the input, are used to denote the statuses of protective relays and circuit breakers in fault section, while proposition neuron $\sigma_{23}$ as the output is used to denote the confidence degree of fault section. The initial values of all input neurons are determined according to Tables III and IV.

The IFSNP systems of lines $L 30$ and $L 29$ can be easily reasoned by using the proposed fuzzy reasoning algorithm. Since $L 30$ and $L 29$ have a similar reasoning procedure, line $L 30$ as an example is illustrated as follow. Initially, $\theta_{0}$ and $\delta_{0}$ can be determined according to the status information of protective relays in the fault situation and Tables II-IV as follows, where $\theta_{0}$ is a 23-dimensional vector and $\delta_{0}$ is a 13-dimensional vector.

$$
\delta_{0}=[\mathbf{0}], \theta_{0}=\left(\begin{array}{c}
{[0.90,0.10]} \\
{[0.90,0.10]} \\
{[0.90,0.10]} \\
{[0.90,0.10]} \\
{[0.25,0.60]} \\
{[0.25,0.60]} \\
\mathbf{0}
\end{array}\right) .
$$

According to fuzzy reasoning algorithm, computing results of each iteration are provided as follows.

For $t=1$,

$$
\delta_{1}=\left(\begin{array}{c}
{[0.81,0.19]} \\
{[0.81,0.19]} \\
{[0.225,0.64]} \\
{[0.225,0.64]} \\
\mathbf{0}
\end{array}\right), \theta_{1}=\left(\begin{array}{c}
\mathbf{0} \\
{[0.81,0.19]} \\
{[0.81,0.19]} \\
{[0.225,0.64]} \\
{[0.225,0.64]} \\
{[0.00,0.90]} \\
{[0.00,0.90]} \\
\mathbf{0}
\end{array}\right) .
$$

For $t=2$,

$$
\delta_{2}=\left(\begin{array}{c}
{[0.81,0.19]} \\
{[0.81,0.19]} \\
{[0.81,0.19]} \\
{[0.225,0.64]} \\
{[0.81,0.19]} \\
{[0.225,0.64]} \\
\mathbf{0}
\end{array}\right), \theta_{2}=\left(\begin{array}{c}
\mathbf{0} \\
{[0.81,0.19]} \\
{[0.81,0.19]} \\
{[0.81,0.19]} \\
{[0.225,0.64]} \\
{[0.81,0.19]} \\
{[0.225,0.64]} \\
\mathbf{0}
\end{array}\right) .
$$

For $t=3$,

$$
\delta_{3}=\left(\begin{array}{c}
\mathbf{0} \\
{[0.81,0.19]}
\end{array}\right), \theta_{3}=\left(\begin{array}{c}
\mathbf{0} \\
{[0.81,0.19]}
\end{array}\right) .
$$

For $t=4$, we have $\delta_{4}=[0]$. Therefore, the halting condition is satisfied and the reasoning procedure ends. Thus, the fuzzy value of output neuron $\delta_{23}$ is [0.81, 0.19]. Based on the judgment condition, $L 30$ is adjudged as a fault section with a confidence level VH.

Similarly, IFSNP system for $L 29$ can be reasoned, and the reasoning result of $L 29$ is $[0.64,0.19]$. Note that 


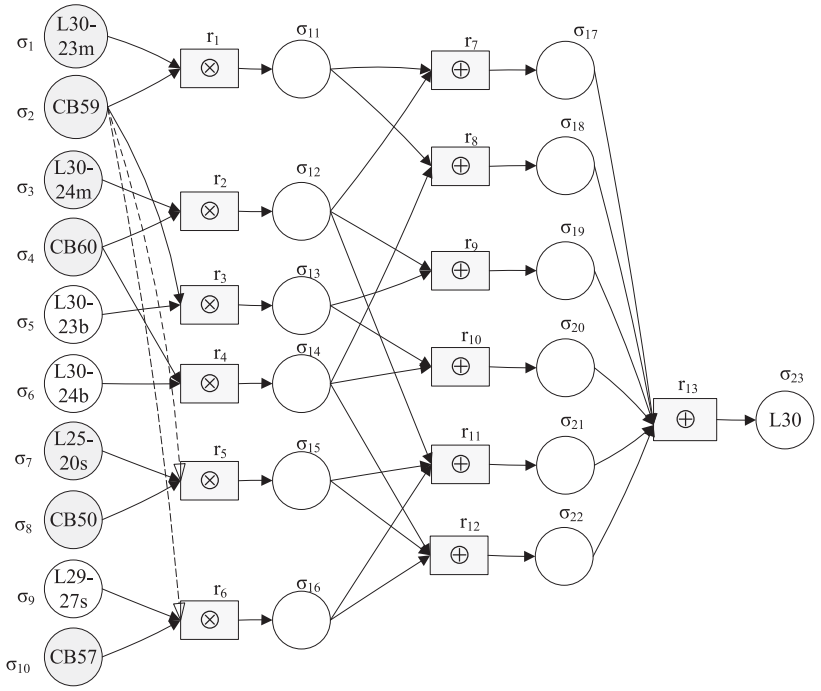

Fig. 8. The fault diagnosis model of line $L 30$ based on IFSNP systems.

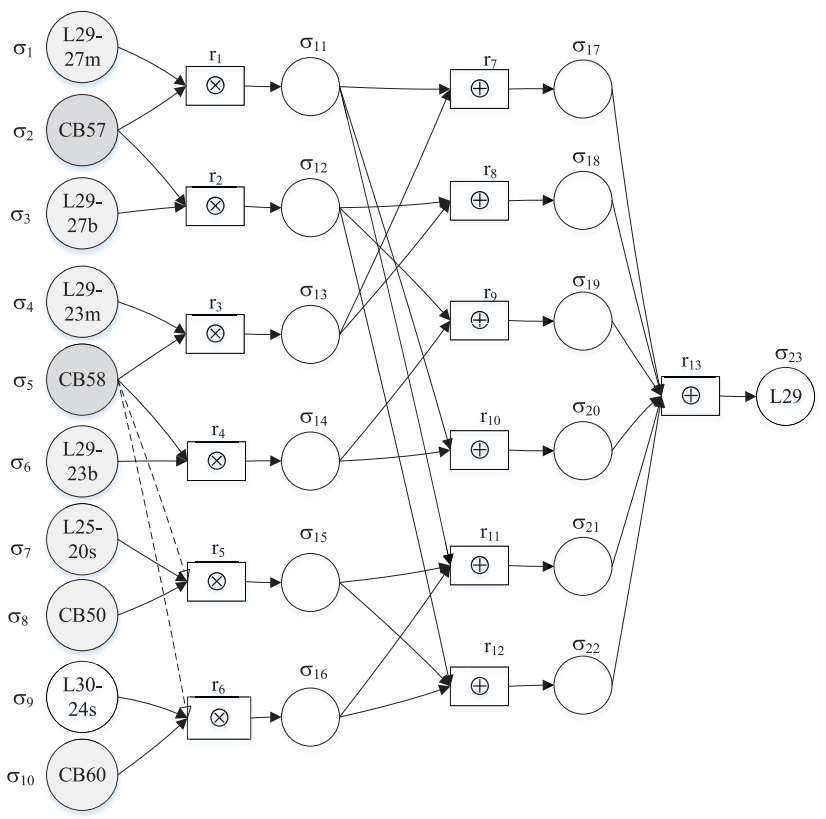

Fig. 9. The fault diagnosis model of line $L 29$ based on IFSNP systems.

$\mathrm{MH}<[0.64,0.19]<\mathrm{H}$ meets. Therefore, $L 29$ is a fault section according to the judgment condition. The diagnostic result of $L 29$ is same to $L 30$ although there is the rejection in sections of $L 29$.

The example indicates that in the case of multiple faults with rejection and incorrect tripping signals the proposed IFSNP systems can accurately diagnose fault sections.

\section{Comparison Analysis With Other Methods}

In the recent, example II has been studied in several literatures, such as fuzzy logic (FL) [10], fuzzy relations (FR) [11], fuzzy graph (FG) [12] and FSNP systems [35]. Chin [10] combined classical fuzzy logic with cause-effect network to deal with the uncertainty in fault diagnosis of power systems. Min et al. [11] presented a fault method based on fuzzy relations, where the relationship between the operated protective
TABLE V

The Comparison Results of the Proposed Method With OTHER METhODS ON EXAMPLE II

\begin{tabular}{|c|c|c|c|c|}
\hline IFSNP systems & FL [10] & FR [11] & FG [12] & FSNP systems [35] \\
\hline L29, L30 & L30 & L30 & L29, L30 & L30 \\
\hline
\end{tabular}

devices and the fault section candidates was modeled and reasoned by fuzzy matrix. Chen [12], fuzzy graph was used to propose a fault diagnosis method. Tu et al. [35], FSNP systems were applied to deal with fault diagnosis problem of power systems. In these four methods, classical fuzzy logic and reasoning mechanism were used to express and handle the uncertainty in fault diagnosis of power systems. In this work, a fault diagnosis problem can be described by a set of fuzzy production rules, and then rule neurons and proposition neurons are used to express the fuzzy rules and the fuzzy propositions in them respectively. Moreover, fault diagnosis is implemented based on the firing mechanism of neurons, and IFNs are used to express the uncertainty in fault diagnosis problems.

The comparison results of the proposed fault diagnosis model based on IFSNP systems with these methods on example II are provided in Table V. It can be observed from Table V that IFSNP systems and FG methods can diagnose the faults $L 29$ and $L 30$, however, FL, FR and FSNP systems can distinguish only the fault L30. More importantly, the proposed fault diagnosis model not only can correctly identify all the fault sections but also provides two measures of each fault section (membership degree and non-membership degree). Thus, IFSNP systems can distinguish a fault section with high confidence level (higher membership degree and lower non-membership degree). In addition, the comparison results of IFSNP systems with FSNP systems indicate that IFN has stronger ability to characterize the uncertainty in fault diagnosis problem of power systems than classical fuzzy number. The comparison demonstrates that fault diagnosis model based on IFSNP systems is effective for fault diagnosis of power systems.

\section{CONCLUSION}

This paper developed IFSNP systems and presented a novel fault diagnosis model based on IFSNP systems for power systems. The IFSNP systems are a kind variant that integrates IFN in SNP systems, therefore, the proposed modeling method is capable of representing uncertain knowledge in fault diagnosis of power systems and dealing with alarm messages from the SCADA system. Moveover, IFSNP systems can more finely and accurately distinguish whether an element is a fault section by providing its membership and non-membership degrees simultaneously. Therefore, IFSNP systems can help the dispatchers more intuitively and effectively to identify all the fault sections. The case studies on a six-bus $69 \mathrm{kV}$ distribution system and a $345 \mathrm{kV}$ transmission system demonstrate that the proposed diagnosis method can effectively and accurately deal with single fault, complex fault and multiple faults with rejection and incorrect tripping signals. The proposed fault diagnosis model requires status information provided by 
SCADA system, so it is unable to handle fault diagnosis if SCADA system is not equipped with power systems. In such an application scenario, this is a limitation of IFSNP systems. In addition, the time between the tripping of breakers during a fault is worth considering because they can provide additional information, especially in the systems where stepped-distance and differential protection (and possibly breaker failure protection) are used. However, the current version of IFSNP systems does not contain time factors, so it can not handle the situation. Our further work is to extend IFSNP systems to discuss fault diagnosis in this situation.

\section{ACKNOWLEDGMENT}

The authors would like to thank the anonymous reviewers for their very insightful and constructive suggestions, which have helped greatly improve the presentation of this paper.

\section{REFERENCES}

[1] C. A. Protopapas, K. P. Psaltiras, and A. V. Machias, "An expert system for substation fault diagnosis and alarm processing," IEEE Trans. Power Del., vol. 6, no. 2, pp. 648-655, Apr. 1991.

[2] H.-J. Lee, B.-S. Ahn, and Y.-M. Park, "A fault diagnosis expert system for distribution substations," IEEE Trans. Power Del., vol. 15, no. 1, pp. 92-97, Jan. 2000.

[3] Y.-C. Huang, "Fault section estimation in power systems using a novel decision support system," IEEE Trans. Power Syst., vol. 17, no. 2, pp. 439-444, May 2002.

[4] S. C. Tan and C. P. Lim, "Application of an adaptive neural network with symbolic rule extraction to fault detection and diagnosis in a power generation plant," IEEE Trans. Energy Convers., vol. 19, no. 2, pp. 369-377, Jun. 2004.

[5] G. Cardoso, J. G. Rolim, and H. H. Zürn, "Application of neural-network modules to electric power system fault section estimation," IEEE Trans. Power Del., vol. 19, no. 3, pp. 1034-1041, Jul. 2004.

[6] D. Thukaram, H. P. Khincha, and H. P. Vijaynarasimha, "Artificial neural network and support vector machine approach for locating faults in radial distribution systems," IEEE Trans. Power Del., vol. 20, no. 2, pp. 710-721, Apr. 2005.

[7] X. N. Lin, S. H. Ke, Z. T. Li, H. L. Weng, and X. H. Han, "A fault diagnosis method of power systems based on improved objective function and genetic algorithm-tabu search," IEEE Trans. Power Del., vol. 25, no. 3, pp. 1268-1274, Jul. 2010.

[8] S.-J. Huang, X.-Z. Liu, W.-F. Su, and T.-C. Ou, "Application of enhanced honey-bee mating optimization algorithm to fault section estimation in power systems," IEEE Trans. Power Del., vol. 28, no. 3, pp. 1944-1951, Jul. 2013.

[9] S.-J. Huang and X.-Z. Liu, "Application of artificial bee colony-based optimization for fault section estimation in power systems," Int. J. Elect. Power Energy Syst., vol. 44, no. 1, pp. 210-218, Jan. 2013.

[10] H.-C. Chin, "Fault section diagnosis of power system using fuzzy logic," IEEE Trans. Power Syst., vol. 18, no. 1, pp. 245-250, Feb. 2003.

[11] S.-W. Min, J.-M. Sohn, J.-K. Park, and K.-H. Kim, "Adaptive fault section estimation using matrix representation with fuzzy relations," IEEE Trans. Power Syst., vol. 19, no. 2, pp. 842-848, May 2004.

[12] W.-H. Chen, "Online fault diagnosis for power transmission networks using fuzzy digraph models," IEEE Trans. Power Del., vol. 27, no. 2, pp. 688-698, Apr. 2012.

[13] J. Sun, S.-Y. Qin, and Y.-H. Song, "Fault diagnosis of electric power systems based on fuzzy Petri nets," IEEE Trans. Power Syst., vol. 19, no. 4, pp. 2053-2059, Nov. 2004.

[14] X. Luo and M. Kezunovic, "Implementing fuzzy reasoning Petri-nets for fault section estimation," IEEE Trans. Power Del., vol. 23, no. 2, pp. 676-685, Apr. 2008.

[15] G. Păun, "Computing with membranes," J. Comput. Syst. Sci., vol. 61, no. 1 , pp. $108-143$, Aug. 2000.

[16] G. Păun, G. Rozenberg, and A. Salomaa, The Oxford Handbook of Membrane Computing. New York, NY, USA: Oxford Univ. Press, 2010.

[17] T. Song, L. Pan, and G. Păun, "Asynchronous spiking neural P systems with local synchronization," Inf. Sci., vol. 219, pp. 197-207, Jan. 2013.
[18] X. Zhang, Y. Liu, B. Luo, and L. Pan, "Computational power of tissue P systems for generating control languages," Inf. Sci., vol. 278, pp. 285-297, Sep. 2014.

[19] G. Zhang, M. Gheorghe, L. Pan, and M. J. Pérez-Jiménez, "Evolutionary membrane computing: A comprehensive survey and new results," Inf. Sci., vol. 279, pp. 528-551, Sep. 2014.

[20] X. Zeng, L. Xu, X. Liu, and L. Pan, "On languages generated by spiking neural P systems with weights," Inf. Sci., vol. 278, pp. 423-433, Sep. 2014.

[21] H. Peng, J. Wang, M. J. Pérez-Jiménez, and A. Riscos-Núñez, "An unsupervised learning algorithm for membrane computing," Inf. Sci., vol. 304, pp. 80-91, May 2015.

[22] H. Peng, J. Wang, P. Shi, A. Riscos-Núñez, and M. J. Pérez-Jiménez, "An automatic clustering algorithm inspired by membrane computing," Pattern Recognit. Lett., vol. 68, pp. 34-40, Dec. 2015.

[23] H. Peng, Y. Jiang, J. Wang, and M. J. Pérez-Jiménez, "Membrane clustering algorithm with hybrid evolutionary mechanisms," J. Softw., vol. 26, no. 5, pp. 1001-1012, May 2015.

[24] J. Wang, P. Shi, and H. Peng, "Membrane computing model for IIR filter design," Inf. Sci., vol. 329, pp. 164-176, Feb. 2016.

[25] H. Peng, J. Wang, P. Shi, M. J. Pérez-Jiménez, and A. Riscos-Núñez, "An extended membrane system with active membranes to solve automatic fuzzy clustering problems," Int. J. Neural Syst., vol. 26, no. 3, pp. 1-17, May 2016.

[26] B. Song, M. J. Pérez-Jiménez, and L. Pan, "An efficient time-free solution to SAT problem by P systems with proteins on membranes," J. Comput. Syst. Sci., vol. 82, no. 6, pp. 1090-1099, Sep. 2016.

[27] L. Pan, G. Păun, and B. Song, "Flat maximal parallelism in P systems with promoters," Theor. Comput. Sci., vol. 623, pp. 83-91, Apr. 2016.

[28] M. Ionescu, G. Păun, and T. Yokomori, "Spiking neural P systems," Fundamenta Informaticae, vol. 71, nos. 2-3, pp. 279-308, Jun. 2006.

[29] L. Q. Pan, J. Wang, and H. J. Hoogeboom, "Spiking neural P systems with astrocytes," Neural Comput., vol. 24, no. 3, pp. 805-825, Mar. 2012.

[30] G. X. Zhang, H. N. Rong, F. Neri, and M. J. Pérez-Jiménez, "An optimization spiking neural $\mathrm{P}$ system for approximately solving combinatorial optimization problems," Int. J. Neural Syst., vol. 24, no. 5, pp. 1-16, Aug. 2014.

[31] J. Wang, L. Zhou, H. Peng, and G. Zhang, "An extended spiking neural P system for fuzzy knowledge representation," Int. J. Innov. Comput. Inf. Control, vol. 7, no. 7A, pp. 3709-3724, Jun. 2011.

[32] J. Wang, P. Shi, H. Peng, M. J. Pérez-Jiménez, and T. Wang, "Weighted fuzzy spiking neural P systems," IEEE Trans. Fuzzy Syst., vol. 21, no. 2, pp. 209-220, Apr. 2013.

[33] H. Peng et al., "Fuzzy reasoning spiking neural $\mathrm{P}$ system for fault diagnosis," Inf. Sci., vol. 235, pp. 106-116, Jun. 2013.

[34] J. Wang and H. Peng, "Adaptive fuzzy spiking neural P systems for fuzzy inference and learning," Int. J. Comput. Math., vol. 90, no. 4, pp. 857-868, Feb. 2013.

[35] M. Tu, J. Wang, H. Peng, and P. Shi, "Application of adaptive fuzzy spiking neural P systems in fault diagnosis of power systems," Chin. J. Electron., vol. 23, no. 1, pp. 87-92, Jan. 2014.

[36] T. Wang et al., "Fault diagnosis of electric power systems based on fuzzy reasoning spiking neural P systems," IEEE Trans. Power Syst., vol. 30, no. 3, pp. 1182-1194, May 2015.

[37] J. Wang, H. Peng, M. Tu, J. M. Pérez-Jiménez, and P. Shi, "A fault diagnosis method of power systems based on an improved adaptive fuzzy spiking neural P systems and PSO algorithms," Chin. J. Electron., vol. 25, no. 2, pp. 320-327, Mar. 2016.

[38] K. T. Atanassov, "Intuitionistic fuzzy sets," Fuzzy Sets Syst., vol. 20, no. 1, pp. 87-96, Aug. 1986.

[39] K. T. Atanassov, Intuitionistic Fuzzy Sets: Theory and Applications. New York, NY, USA: Physica-Verlag, 1999.

[40] Y. He, H. Chen, Z. He, and L. Zhou, "Multi-attribute decision making based on neutral averaging operators for intuitionistic fuzzy information," Appl. Soft Comput., vol. 27, pp. 64-76, Feb. 2015. 